


\section{Homologous recombination:}

\section{Single-molecule experiments and their lessons for the in vivo situation}

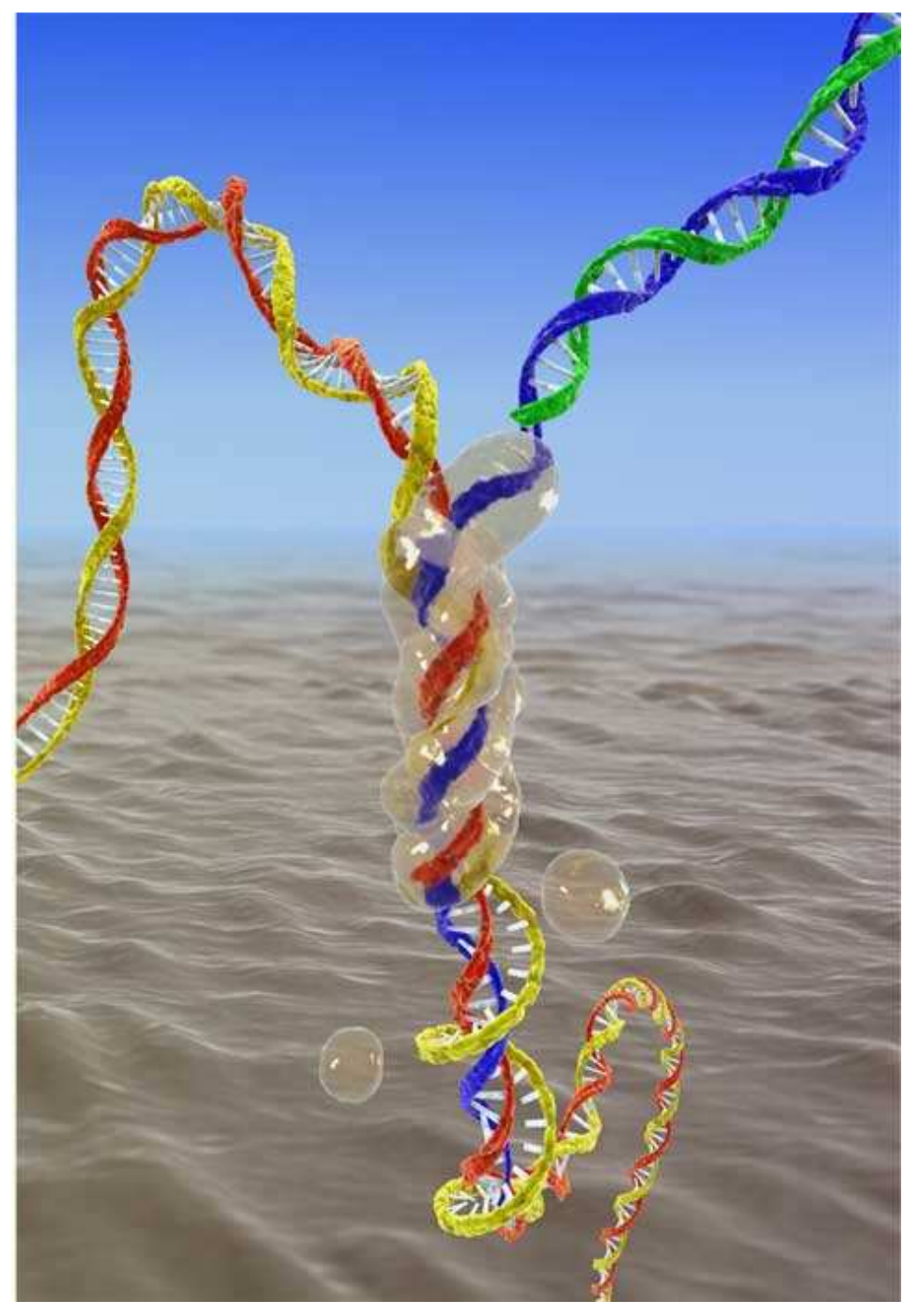

Literature thesis

Mariella Franker, 1465465

Biomolecular Sciences, Cell Biology June 2009, 9 ECTS 


\section{Summary}

Homologous recombination is an essential DNA repair mechanism in both prokaryotes and eukaryotes. It especially plays an important role in the repair of broken or stalled replication forks and is vital for proper chromosome segregation and immunoglobulin diversity. The main event in homologous recombination is the formation of a nucleoprotein filament by RecA-like proteins. Assembly of this filament is the rate-limiting step in recombination and it mediates subsequent stages of repair. Single-molecule experiments have given great insights into the physical mechanism and function of the nucleoprotein filament. In vivo, however, many recombination mediators are involved in the processes and various complex pathways are activated. 


\section{Introduction}

DNA damage is caused by various internal and external factors. Reactive oxygen species that are continuously produced by the body; DNA nucleases; viral infections; toxins; and radiation all threaten genomic integrity. Of the different types of DNA damage, double-strand breaks (DSBs) belong to the most severe and the cell employs two main pathways to repair them: nonhomologous end-joining and homologous recombination. Non-homologous end-joining (NHEJ) simply fuses the two ends of the DSB together. In some cases a few nucleotides are removed or added at each end of the break to facilitate ligation and this can compromise gene stability. However, breaks in a single parental duplex are efficiently repaired by NHEJ in all stages of the cell cycle ${ }^{1}$. Homologous recombination (HR) uses the homologous sister chromatid to repair broken strands and all genetic information is retained during this process. HR is commonly seen during and shortly after replication when the sister chromatid is still available (S and G2 phase) and especially plays an important role in the repair of broken replication forks. During meiosis, specialized endonucleases purposely induce DSBs and homologous recombination is essential for proper chromosome segregation and immunoglobulin diversity $(\mathrm{V}(\mathrm{D}) \mathrm{J} \text { switching })^{2,3}$. The most important phase in homologous recombination is binding of RecA-like recombinase proteins to ssDNA overhangs created around the double strand break. These proteins, RecA in prokaryotes and Rad51 in eukaryotes, form a nucleoprotein filament on ssDNA that mediates further steps of homologous recombination. RecA and Rad51 have similar properties and catalyze the same reactions during HR; however some important functional differences have been discussed ${ }^{4}$. Here, we will mainly focus on the eukaryotic Rad51. In vitro, isolated Rad51 is capable of catalyzing homologous recombination; however, in vivo many recombination mediators are involved in the process. One important group of proteins that regulates many, if not all, stages of HR is the Rad52 epistasis group ${ }^{5}$.

The past few decades, single-molecule experiments, such as single-molecule fluorescence, scanning force microscopy (SFM), fluorescence resonance energy transfer (FRET), optical tweezers (OT), and magnetic tweezers (MT), have changed the way biological systems are being studies ${ }^{6-8}$. However, single-molecule experiments generally cannot study molecular processes in vivo. Highly artificial conditions are created during these experiments and one wonders how relevant the findings of single-molecule experiments are for the in vivo situation.

This review offers an overview of the mechanism of homologous recombination, especially the role of Rad51 therein. Furthermore, homologous recombination in vivo is considered in more detail followed by a short discussion on the implications of single-molecule experiments. 


\section{General mechanism}

Homologous recombination includes three major steps: (i) initiation and nucleoprotein filament assembly, (ii) homology search and DNA exchange (strand invasion), (iii) filament disassembly and branch resolution. The exact mechanism of homologous recombination is not yet fully understood and currently two primary models have been described: the double-strand breakrepair model (DSBR) and the synthesis-dependent strand annealing (SDSA). These models were first developed to explain recombination products found during meiosis and mitosis in bacteria and yeast, but it is still unclear whether they operate separately or in conjunction ${ }^{5,} 9$. Both models share the first few steps of the process including initiation and strand invasion (fig. 1a). Shortly after detection of a double-strand break, the ends around the damage site are resected by exonucleases in a 5' to 3' direction. This produces single-stranded DNA (ssDNA) overhangs with exposed 3'-OH ends. Recombinase proteins, such as RecA in prokaryotes and Rad51 in eukaryotes, bind to these ssDNA ends and form the nucleoprotein filament or presynaptic filament. The nucleoprotein filament searches the sister chromatid for a homologous region and invades the duplex DNA. Two recombinases, Rad51 and Dmc1, have been identified in eukaryotic cells. Rad51 is active in both somatic and meiotic cells. Dmc1 is only active during meiosis and acts in conjunction with Rad51 $1^{5}$. During strand invasion, a D-loop structure is formed. Initially, the invading strand and the homologous strand are paired but not yet topologically linked (paranemic joint). Subsequently, a plectonemic joint is formed in which the duplex DNA and the invading strand are bound by Watson-Crick base pairing and are topologically intertwined. DNA polymerase extends the 3 '-end of the invading strand while in the D-loop formation. In SDSA (fig. 1b) the D loop is unwound and the now extended invading strand anneals with the other double strand break end. DNA synthesis and ligation complete the reaction. SDSA results in only non-crossover products that have not undergone any information exchange between chromosomes. In DSBR (fig. 1c) the second resected double-strand break end is captured and annealed to the D loop to form two Holliday junctions (HJ). At this stage, branch migration can occur during which the cross-over junctions move along the DNA and lengthen the newly formed heteroduplex region. The crossed strands are cut by specialized endonucleases resulting in either crossover or non-crossover products. 


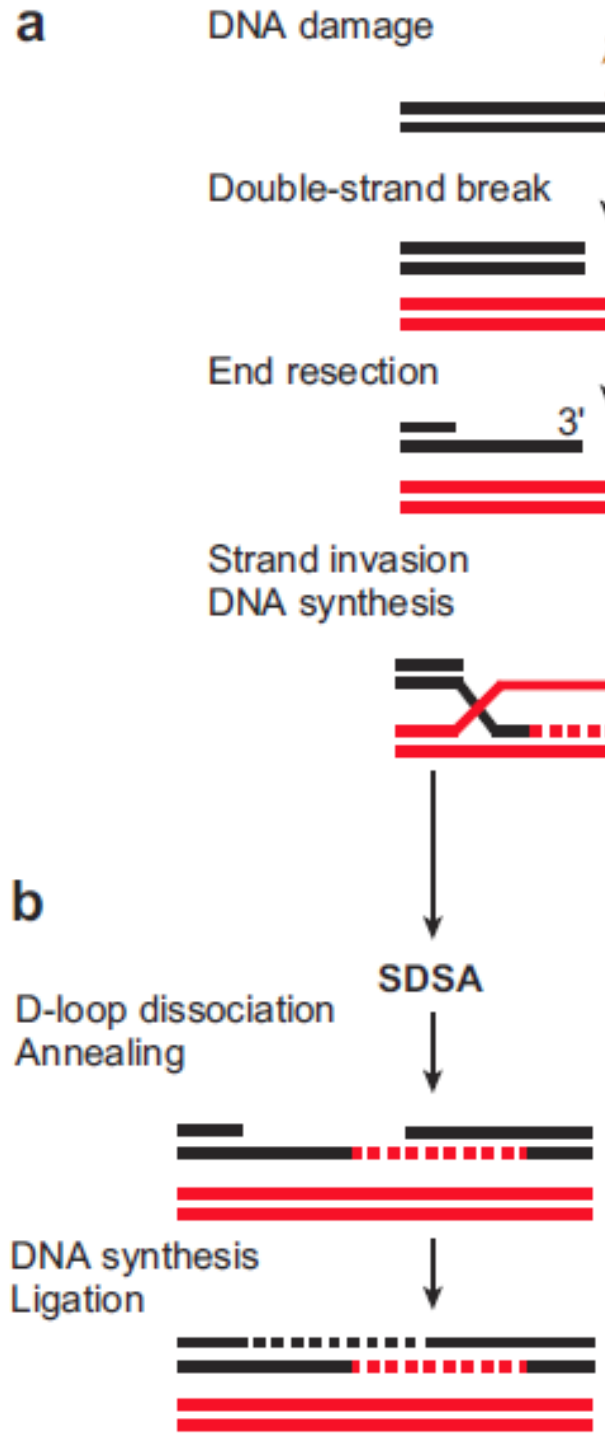

Noncrossover
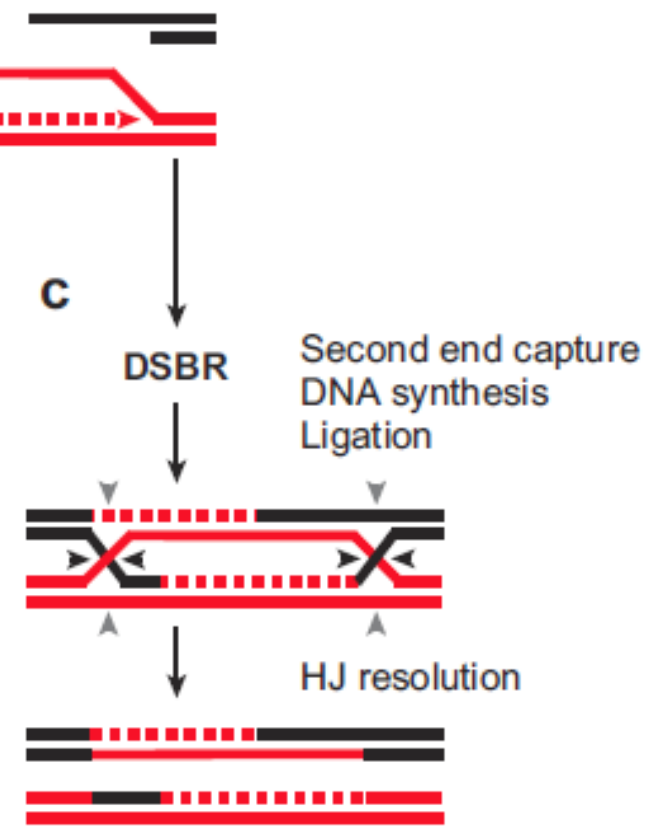

Noncrossover

or

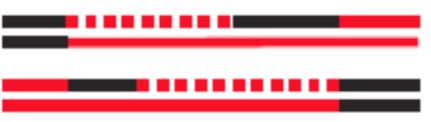

Crossover

Figure 1 Double strand break repair mechanism ${ }^{b}$. a) Initiation and strand invasion. HR is initiated by resection of double strand break (DSB) ends by exonucleases. The nucleoprotein filament, consisting of DNA-bound Rad51 or RecA proteins, searches the sister chromatid for a homologous region and facilitates $D$-loop formation and strand invasion. b) SDSA pathway. The D loop is unwound and the invading strand anneals to the other DSB end. DNA synsthesis and ligation yield non-crossover products. c) DSBR pathway. The second DSB end is captured to form a heteroduplex containing two Holliday junctions $(\mathrm{HJ})$. Branch migration can occur during this intermediate state. Endonucleases cut HJs (small arrowheads) and yield crossover or non-crossover products. 


\section{Nucleoprotein filament: structure and function}

The nucleoprotein filament is the driving force behind homologous recombination and filament assembly and disassembly are highly dynamic processes. Mutations in functional regions of Rad51 decrease efficiency of double strand break repair and increase lethality ${ }^{10,}{ }^{11}$. In vitro, isolated Rad51 is capable of catalyzing strand exchange in the absence of recombination mediators. The structure of recombinases is highly conserved, indicating a strong structurefunction relation.

\section{Monomer}

The structure of the Rad51 monomer was determined in a Rad51-BRCA2 complex ${ }^{12}$. The wellknown tumor suppressor BRCA2 is commonly suppressed in breast cancer and has been identified as an important recombination mediator ${ }^{13}$. Figure 2 a shows BRC repeat 4 (BRC4) of BRCA2 interacting with the subunit-subunit interface of Rad51. The core domain of recombinases is highly conserved between species (fig. 2c) and shows strong homology with $F_{1^{-}}$ ATPase and DNA helicases ${ }^{14}$. Mutagenesis experiments have determined various functional regions in the core domain ${ }^{15,16}$. Figure $2 b$ shows the oligomerization interface or subunit-subunit interface in white. Interestingly, aromatic residues (blue) are mainly found on the inner surface of the filament (top image) and on the subunit-subunit interface. A walker motif or $\mathrm{P}$ loop present in the structure coordinates binding of ATP and $\mathrm{Mg}^{2+}$-ions. The nucleotide binding site (shown in green) is found between the monomers. ATP binding is essential for filament assembly and strand invasion, but ATP hydrolysis promotes filament disassembly. Crystal structures of the L1 and $L 2$ loops (orange and red, respectively) are not observed in the monomer because of their flexibility. RecA contains a large C-terminal region, whereas Rad51 contains a $\mathrm{n}$ extended $\mathrm{N}$ terminal region (fig. 2c). These loops and terminal domains are involved in DNA binding ${ }^{17}$ and may also play a role in oligomerization and interaction with recombination mediators ${ }^{18}$.

\section{Filament assembly}

The nucleoprotein filament elongates and partly unwinds DNA, extending it to $\sim 150 \%$ of its original length. The resulting extension and tension are necessary for access of the recombination machinery to the homologous strand and are important for homology search and strand invasion. The nucleoprotein filament consists of a right-handed helical protein filament with $\sim 6$ monomers per turn and underwound DNA of $\sim 19$ bp per turn and can span thousands of base pairs ${ }^{29}$. 

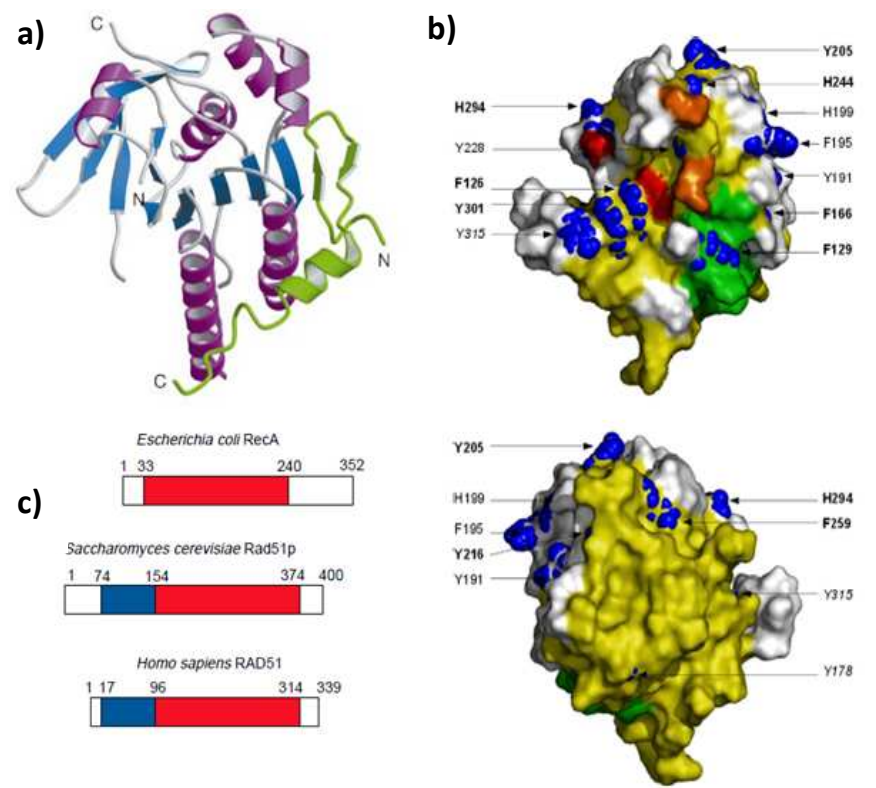

Figure 2 a) Crystal structure of human Rad51-BRCA2 complex ${ }^{12}$. Ribbon representation of the central domain of Rad51 ( $\alpha$-helices in magenta and $\beta$-strands in blue) interacting with BRC4 repeat of BRCA2 (green). b) Functional regions of core domain of human Rad51 as seen from the inside (top) and the outside of the filament (bottom) ${ }^{15}$. Aromatic residues are shown in blue; subunitsubunit interface in white; nucleotide binding site in green; and ends of L1 and L2 loop in orange and red respectively. Note that top left in b) is bottom right in a). c) Sequence comparison between RecA and Rad51 ${ }^{29}$. Conserved core region is shown in red and eukaryotic $\mathrm{N}$-terminal region is shown in blue. A large C-terminal region is found in RecA but not in Rad51.

RecA and Rad51 are capable of forming filaments on both ssDNA and dsDNA in the presence of ATP or an ATP analog and strong allosteric coupling between ATP binding, DNA binding and ATPase activity is observed ${ }^{15}$. Binding affinity of RecA is significantly higher for SsDNA, while Rad51 has similar affinity for ssDNA and dsDNA ${ }^{9,14}$.

Formation of the nucleoprotein filament is the rate-limiting step in homologous recombination. Fully active filaments require the presence of ATP, $\mathrm{Mg}^{2+}$-ions and salt (e.g. $\mathrm{NaCl}, \mathrm{KCl}$ ). Varying the nature and concentrations of these cofactors can greatly influence the dynamics of the filament. Inactive filaments bound to ADP are relatively condensed (pitch $\sim 65-85 \AA$ ) compared to the active filament (pitch $\sim 83-130 \AA$ ). Filaments bound to the non-hydrolizable ATP analog ADP-

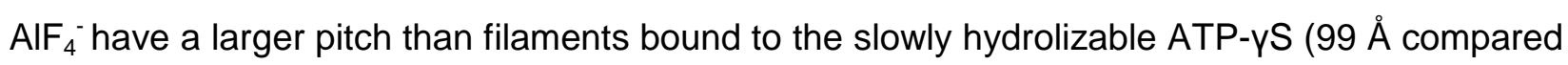
to $76 \AA)^{19}$. Addition of $\mathrm{Ca}^{2+}$ instead of $\mathrm{Mg}^{2+}$ inhibits ATP hydrolysis and stabilizes the filament and high salt concentrations $(\geq 100-150 \mathrm{mM})$ inhibit nucleation ${ }^{20,21}$.

Filament assembly can roughly be divided into two stages: nucleation and growth. Nucleation events are easily controlled by altering the nature of the nucleotide cofactor and protein or salt 
concentrations, but filament growth is less sensitive to these factors. This suggests that control of nucleation might be an important regulatory step in vivo ${ }^{20,22}$. Distinguishing experimentally between filament extension and additional nucleation events is difficult, and there is some debate about the size of the actual DNA-binding unit. Single-molecule FRET experiments allowed determination of end-to-end distance as a measure of RecA-induced DNA extension (see fig. 3a). These experiments indicate that the binding unit is most likely multimeric ${ }^{23}$. Comparable results were found for Rad51 with magnetic tweezer experiments and Monte Carlo simulations $^{24}$. In the magnetic tweezer setup, DNA molecules attached to a paramagnetic bead can be stretched or twisted with magnets above the sample. When Rad51 is added, the DNA is elongated and video microscopy is used to track bead position and determine the end-to-end distance (fig. 3b). A more direct approach using fluorescently labeled Rad51 proteins and optical trapping allowed direct imaging of DNA-bound proteins. Nanometer polystyrene beads are trapped using a high powered laser and a single DNA molecule is bound to the beads via biotinstreptavidin links. Binding of fluorescently labeled Rad51 proteins to the DNA molecule is directly observed (fig. 3c). Specially designed flow cell systems allow quick exchange of buffer conditions.

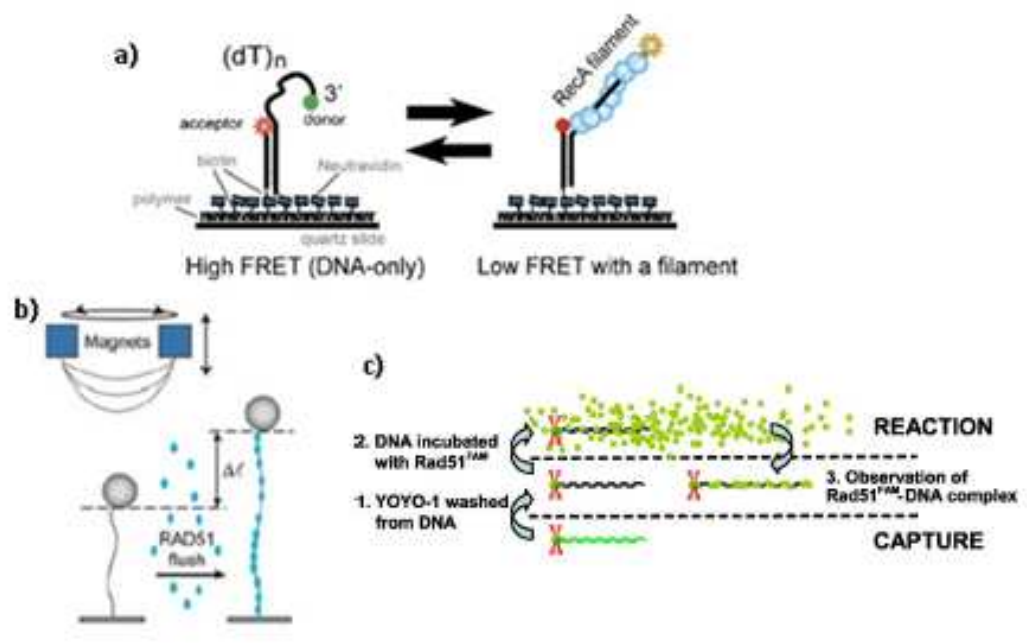

Figure 3 Single molecule approaches commonly used in nucleic acids research a) FRET analysis of RecA filament dynamics ${ }^{23}$. A biotinylated dsDNA with a 3' ssDNA tail was immobilized on a glass surface. Donor and receptor fluorophores were attached to the ssDNA end and at the ss-dsDNA junction. Naked DNA is flexible and gives high FRET signal. When RecA binds, the DNA is extended and the fluorophores are separated, resulting in a decrease in FRET signal. b) Magnetic tweezer approach ${ }^{24}$. A DNA molecule was attached to a glass surface and stretched using magnets above the sample holder. Binding of Rad51 increases the end-to-end distance and can be observed using video microscopy and image analysis. c) Single-molecule fluorescence $^{21}$. Streptavidin covered beads are held in place by optical traps. A biotinylated DNA molecule is bound to the bead and stretched by flow force. A specialized flow cell system allows quick buffer exchanges. Fluorescently labeled Rad51 binds to DNA and is imaged directly. 
The setup shown here uses a single bead per molecule and the DNA is stretched by flow force. A different setup where a single DNA molecule is spanned between two beads allows yet unparalleled control of forces. Large forces of up to $\sim 100 \mathrm{pN}$ can be applied to the DNA molecule ${ }^{25}$. Experiments done with these single-molecule approaches indicate that nucleation of the nucleoprotein filament most likely occurs via binding of 3-5 monomers ${ }^{24,24}$.

After nucleation, the filament rapidly extends via binding of additional protomers. Independent studies using FRET and single-molecule fluorescence show that filament extension likely occurs by binding of dimers or single monomers ${ }^{21,23}$. Monte Carlo simulations, however, predict growth by pentamers ${ }^{24}$. The level of cooperativity during filament formation is determined by the relative rates of nucleation and extension and these rates influence the binding unit size that can be resolved experimentally. RecA growth rates are much higher than nucleation rates $\left(10^{9}\right.$ fold), resulting in extremely cooperative filament formation. Once nucleated, the RecA filament quickly elongates and a continuous filament is formed. Extension rates of Rad51 are only slightly higher than nucleation rates $\left(10^{2}\right.$ fold) and consequently Rad51 forms smaller filament patches with short gaps of bare DNA in between. Images of Rad51 filaments on dsDNA obtained with scanning force microscopy (SFM) frequently have kinks, indicating the flexible 'hinge' regions of bare DNA within the filament ${ }^{22}$. These kinks were not observed in RecA filaments formed under similar conditions (fig. 4a). Epi-fluorescence images taken from fluorescently labeled Rad51 proteins also show the characteristic filament patches (fig. 4b). Many experiments describe unidirectional 5' to $3^{\prime}$ growth of the nucleoprotein filaments ${ }^{9,}{ }^{14}$, but in vitro experiments with RecA have found slow extension in 3' to 5' direction and bidirectional growth may occur at different rates in vivo as well ${ }^{20}$. A kymograph (shown in fig. 4d, left) depicts fluorescence intensity along the DNA molecule in time ${ }^{22}$. The filament is stable in the presence of $\mathrm{Ca}^{2+}$ and ATP. AT $\mathrm{t}=15 \mathrm{~min}, \mathrm{Mg}^{2+}$ is added. Clearly, the molecule shrinks and the fluorescence intensity decreases as the filament disassembles. The graph on the right shows the integrated intensity in time in presence of $\mathrm{Ca}^{2+}$ and $\mathrm{Mg}^{2+}$. Figure 4c shows an electron-microscope image of human Rad51 filament formed on dsDNA. The blotchy appearance of the image is due to the helical structure of the filament. DNA strands are bound on the inside of the helix along the filament axis. Here, a largely continuous filament is formed, which is a-typical for Rad51. However, protein concentration used to obtain this image were quite high $(6 \mu \mathrm{M}$, compared to 1-2.5 $\mu \mathrm{M}$ used for fluorescence and SFM images) ${ }^{26}$. The crystal structure of Rad51 bound to ssDNA was determined using a yeast gain-of-function mutant ${ }^{18}$ (fig. 4e). This mutant had increased DNAbinding affinity but unaltered ATPase activity and was fully functional in vivo. Interestingly, the 
structure revealed alternating monomers with slightly different interfaces. Similar asymmetry in filaments is also observed in some helicases and may be important for unwinding properties of Rad51. As with these helicases, sequential ATP binding and hydrolysis may facilitate translocation of the filament along the $D N A^{18}$.

a)

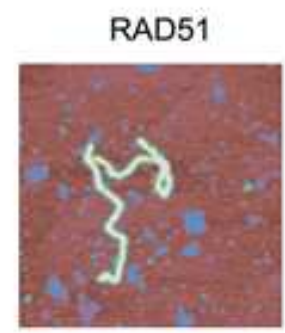

d)

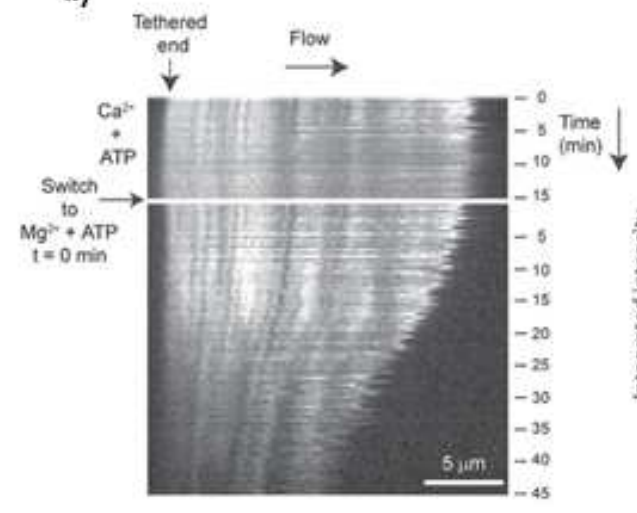

$\operatorname{Rec} A$

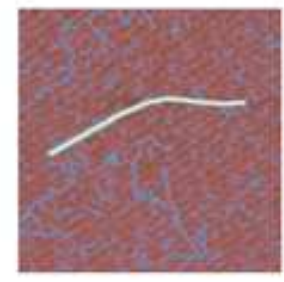

b)
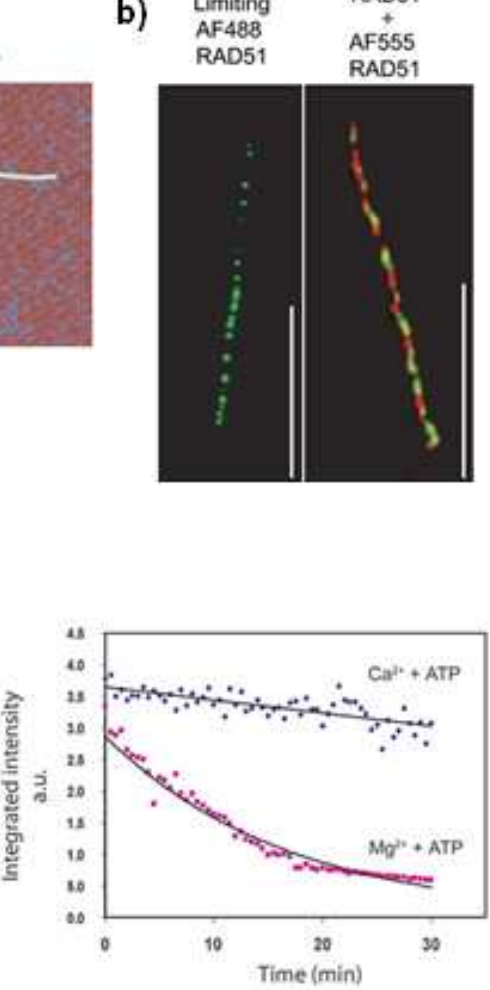

c)

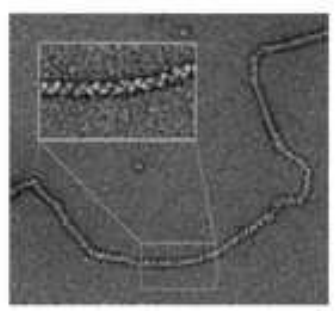

e)

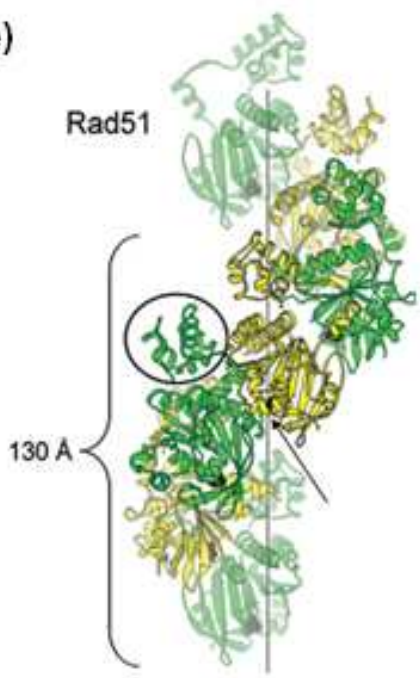

Figure 4 a) Scanning force microscopy (SFM) image of nucleoprotein filament ${ }^{22}$. Rad51 filaments were formed on dsDNA in the presence of ATP and $\mathrm{CaCl}_{2}$ (left). RecA filaments were formed on the same DNA substrate in the presence of ATP- $y$-S and $\mathrm{MgCl}_{2}$. Images are $1 \mu \mathrm{m} \times 1 \mu \mathrm{m}$. b) Fluorescently labeled Rad51 filament formed on dsDNA $^{22}$. dsDNA was incubated with limiting amounts of fluorescently labeled Rad51 in the presence of ATP and $\mathrm{CaCl}_{2}$ and nonspecifically attached to a glass surface. Right panel: Incubation with Rad51-Alexa488 followed by incubation with Rad51-Alexa555. Images were taken with epi-fluorescence microscope; white bars are $10 \mu \mathrm{m}$ markers. c) Electron-microscope image of Rad51 filament $^{29}$. Human Rad51 nucleoprotein filament formed on dsDNA and stained with uranyl acetate. d) Kymograph of type-lapse movie ${ }^{22}$. Behavior of human Rad51 filament formed on dsDNA in the presence of $\mathrm{CaCl}_{2} / \mathrm{ATP}$ for $15 \mathrm{~min}$ and in the presence of $\mathrm{MgCl}_{2} / \mathrm{ATP}$ for $45 \mathrm{~min}$. e) Crystal structure of S. cerevisiae Rad51 filament ${ }^{18}$. A gain-of-function mutant was discovered in a screen for alleles that bypass requirement of important recombination mediators. This mutant had increased DNA-binding affinity but unaltered ATPase activity and was fully functional in vivo. Crystals were grown in the presence of ssDNA and ATP-yS. DNA is bound along the filament axis (black line). 


\section{Strand invasion and disassembly}

The nucleoprotein filament invades the sister chromatid and searches for a homologous sequence. How homology search occurs is still a mystery: how is it possible that the active filament finds the homologous sequence among the vast stretches of DNA inside the nucleus? Several models have been proposed for this mechanism including random collisions between filament and duplex DNA, 1D random walk and unidirectional diffusion along DNA molecules ${ }^{27}$. Random collisions in 3D allow relatively quick sampling over large spaces. An energy barrier to dissociate and reassociate onto the DNA-molecule must be overcome. 1D random walk could accelerate diffusion over short distances by circumventing this dissociation-association activation energy. A slight asymmetry observed in the crystal structure of the Rad51 filament may facilitate unidirectional movement by sequential ATP binding and hydrolysis. This is also seen in some translocating helicases, as mentioned in the previous section. Experiments done on Rad51 found no evidence of translocation ${ }^{22,27}$. One group using total internal reflection fluorescence (TIRF) showed long-distance 1D diffusion of Rad51 along DNA molecules, however, the protein was likely in an inactive conformation and the observed behavior may not be biologically relevant ${ }^{28}$. RecA, but not Rad51, has a motor function that is capable of rotating DNA upon ATP hydrolysis. Using this motor activity RecA is capable of bypassing heterologous regions of up to $100 \mathrm{bp}$, while Rad51 cannot bypass regions $>9 \mathrm{bp}$ heterology. ATP hydrolysis by RecA is $\sim 40$ fold higher than Rad51 and bypass reaction is inhibited when ATP hydrolysis is blocked $^{29}, 30$. Contrary to RecA, Rad51 forms a discontinuous filament consisting of many small patches along the DNA. This greatly increases flexibility of the filament. Magnetic tweezer experiments suggest that a very short region of the nucleoprotein filament interacts with the duplex DNA at any one time (only $\sim 90 \mathrm{bp})^{31}$. Accordingly, the Rad51 filament may contain multiple active sites that simultaneously search for homology. This can compensate for the lack of translocation and motor function and increase efficiency during homology search. It is believed that, during homology search, the duplex DNA is first 'sampled' and aligned by forming a not yet topologically linked paranemic joint. This process may involve base flipping, which is the rotation of bases out of their stacked conformation in order to form transient interactions. Since the less stable A-T bond is easily disrupted, a sufficiently high A:T-content is essential for homology recognition and subsequent strand exchange ${ }^{32}$.

Once the homologous sequence has been located, the DNA regions are aligned and strand invasion can take place. Few studies have been done on strand exchange by Rad51, but several models have been proposed for $\operatorname{Rec}^{30}$ (fig. 5). In one model, RecA rotates two parallel 
oriented DNA molecules around each other's axis (fig. 5a). One DNA molecule is bound along the filament axis and the other molecule is bound on the outside of the filament. This model is consistent with the experimentally determined rates of branch movement during strand invasion and ATP hydrolysis and explains bypass of large regions of heterology. However, no DNA binding domains have been identified on the outside of the filament. Rad51 proteins lack the motor function which is essential in this model. The second model couples strand exchange with filament disassembly (fig. 6b). ATP hydrolysis causes a conformational change that allows protomer release. Rates of filament disassembly determined in bulk experiments are similar to branch progression during strand exchange. The third model proposes that ATP hydrolysis is needed for protein redistribution, to fill discontinuities in the filament, and for filament disassembly. Strand exchange does not require ATP and the filament merely has a DNA-pairing function (fig. 6c).

To complete the reaction, the nucleoprotein filament must dissociate and allow other proteins access to the DNA. Dissociation is generally believed to occur from one end of the filament in a 5'-3' direction, similar to assembly. It is still unclear if dissociation is somehow coordinated between monomers by synchronized ATP activity. A study that combined single-molecule fluorescence with optical tweezers showed that high tension in Rad51-bound dsDNA stabilizes the filament and inhibits disassembly ${ }^{33}$. Quantitive analysis of disassembly traces revealed that disassembly occurs in short bursts of multiple monomers. ADP-bound monomers loose binding affinity for DNA but are held in place by bonds with adjacent monomers. When ATP is hydrolyzed at the filament end, the terminal Rad51 dissociates together with neighboring ADP. bound monomers. Disassembly transiently pauses when an ATP-bound monomer is encountered.

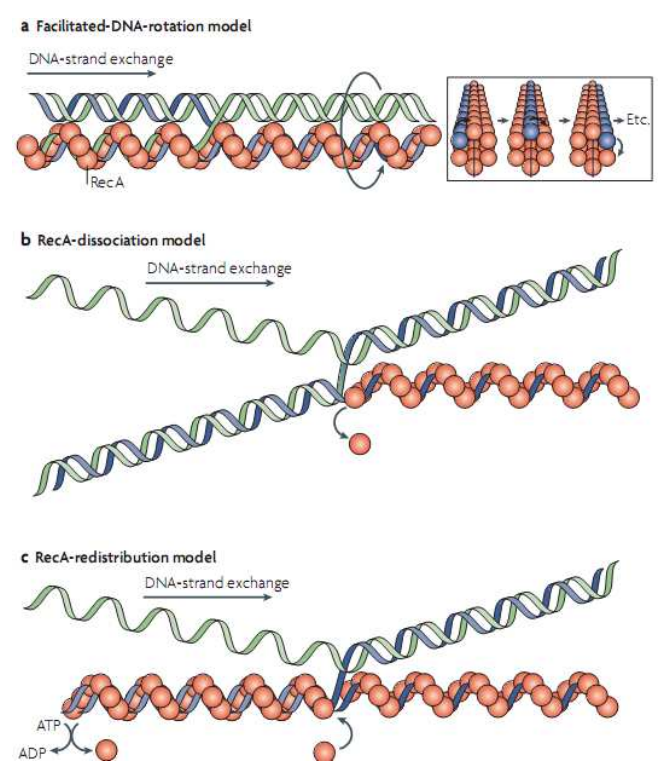

Figure 5 RecA strand invasion models 30 . a) Facilitated DNA-rotation model. Motor function of RecA is essential in this model. The filament rotates two DNA molecules, one bound along the filament axis and the other bound on the outside of the filament, around each other's axis. b) Dissociation model. Filament disassembly is triggered by ATP hydrolysis and is coupled to strand exchange. c) Redistribution model. ATP hydrolysis is necessary to fill discontinuities in the filament and for filament disassembly. Strand exchange itself does not require ATP and the filament merely has a DNA-pairing function. 


\section{In vivo situation: important players and interactions}

Many recombination mediators are involved in homologous recombination in vivo. One important group of proteins that regulates these various stages is the Rad52 epistasis group. This group includes Rad50; Rad51; Rad52; Rad54; Rad55-Rad57 in yeast or Rad51B-D, XRCC2 and XRCC3 in human; Rad59; Rdh54 in yeast or Rad54B in human; Mre11; Xrs2 or Nbs1 in human; and Hop2-Mnd1.

\section{Initiation}

Prior to homologous recombination, various recombination mediators prepare the DNA and recruit or activate the homologous recombination repair proteins. Immediately after DNA damage, double strand break (DSB) ends are recognized by the $M R(X) N$ complex that serves as a DNA-damage sensing unit. Three different proteins are found in this complex: Mre11, Rad50, and Xrs2/ Nbs1, and each plays a specific role in the initiation process. Mre11 has nuclease activity in vitro and recruits other nucleases to the DNA damage site. Mutagenesis studies have shown that it is essential for efficient resection of the DSB 5'-end ${ }^{34}$. Rad50 has an extended coiled-coil structure that spans the DSB and keeps both ends of the break close together ${ }^{35}$. Keeping the DNA strands in close proximity is vital for proper repair, especially in vivo. The role of Xrs2 (Nbs1 in human) is less well defined. Experiments have shown that it is important for targeting the $\mathrm{MR}(\mathrm{X}) \mathrm{N}$ complex to DNA and activation of protein kinases. ATM and the closely related ATR are important transducer kinases recruited and activated by $M R(X) N$. These kinases play a central role in activating the checkpoint response and cell cycle arrest, and once activated recruit various mediator and effector kinases and initiate complex phosphorylation pathways in the cell ${ }^{36,37}$.

Additional pathways are quickly activated by $M R(X) N$ signaling, such as chromatin decondensation to allow access of repair proteins to the damage site, and chromatin modifications to further increase the DNA damage signal. Phosphorylated H2AX histones $(\mathrm{y} 2 \mathrm{AX})$ are found in extending regions on both sides of double strand breaks and assist in assembly of large cohesion complexes around the double strand break to keep the sister chromatid in close proximity ${ }^{38,39}$.

\section{Nucleoprotein filament and strand invasion}

Immediately after resection of the ends around the double strand break, high affinity singlestrand binding proteins SSBs and RPAs (replication protein A) bind the exposed ssDNA and remove any secondary structure of the ssDNA. These proteins can both inhibit and stimulate 
filament formation. Rad51 cannot directly bind RPA-coated SSDNA, but is recruited to the damage site by Rad52 that first removes the RPAs from the ssDNA. RPAs stimulate filament formation by targeting Rad52 and by preventing binding of other, stronger inhibitory proteins ${ }^{5}$. Furthermore, Rad52 plays a role in second-end capture in DSBR pathway and promotes annealing of naked or RPA-covered ssDNA ${ }^{40}$. Rad51 paralogues Rad55 and Rad57 (Rad51B and Rad51C in human) are found as heterodimers and help stabilize already assembled regions of nucleoprotein filament. Studies show that Rad55-Rad57 complex promotes the strand exchange activity of Rad51 41,42 . Hop2-Mnd1 heterodimer preferably binds dsDNA and has highest affinity for branched DNA. It stimulates recombinase activity by stabilizing the nucleoprotein filament and assists in capture of the duplex DNA and formation of the D loop ${ }^{43}$.

The well-known tumor suppressor BRCA2 has been identified as an important regulator in $\mathrm{HR}^{13}$. Although BRCA2 orthologues vary greatly in size, all contain at least one BRC repeat and many contain a structurally distinct C-terminal motif (CTRB domain) capable of interacting with Rad51 ${ }^{5}$. BRC repeats bind the monomeric form of Rad51 such that the Rad51-binding interface is left exposed, which may initiate nucleation and facilitate oligomerization. The CTRB domain binds the oligomeric form of Rad51 and assists in filament assembly. BRCA2 deficiency leads to severe defects in double strand break repair in various cell lines ${ }^{44}$.

\section{Branch migration and resolution}

DNA helicases facilitate branch migration by unwinding duplex DNA and forcing the Holliday junctions along the molecule. Moreover, helicases play an important role in inhibiting undesired recombination events by disrupting the nucleoprotein filament or influencing the formation of crossover products. Recombination between repetitive DNA sequences or formation of improper crossover products can lead to loss of heterozygosity and other deleterious mutations ${ }^{45}$. Topoisomerases and resolvases resolve HJs and allow proper chromosome segregation. Cutting of Holliday junctions can produce both crossover and non-crossover products (see figure 1), but some resolvases, such as Mus81 complex, specifically cleave HJs to yield only crossover products $^{46}$.

Epistatis group member Rad54 has multiple roles in homologous recombination. It is a member of the swi/snf family and plays an important role in chromatin remodeling during the initiation phase. Rad54 translocates on dsDNA and induces topological changes. These interactions may be important in all stages of homologous recombination including homology search, joint molecule invasion, resolution of Holliday junctions, and dissociation of the nucleoprotein filament $^{47}$. It is known that DNA tension plays an important role in Rad51 dissociation. The Rad51-filament is stabilized by high tension and dissociation increases when tension is 
released $^{33}$. Forces induced by Rad54 may play a role in preventing non-specific binding of Rad51 to bulk chromatin. Figure 6 presents an overview of important proteins involved in homologous recombination.

Initiation
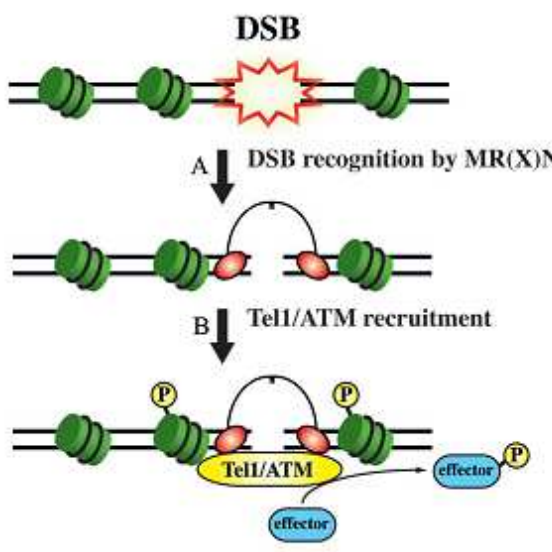

Tel1/ATM checkpoint activation
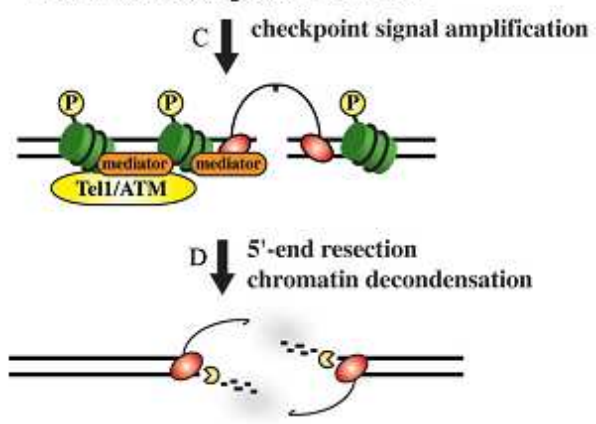

E RPA binding
Strand invasion

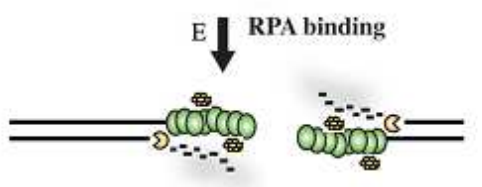

F Rad51-filament formation
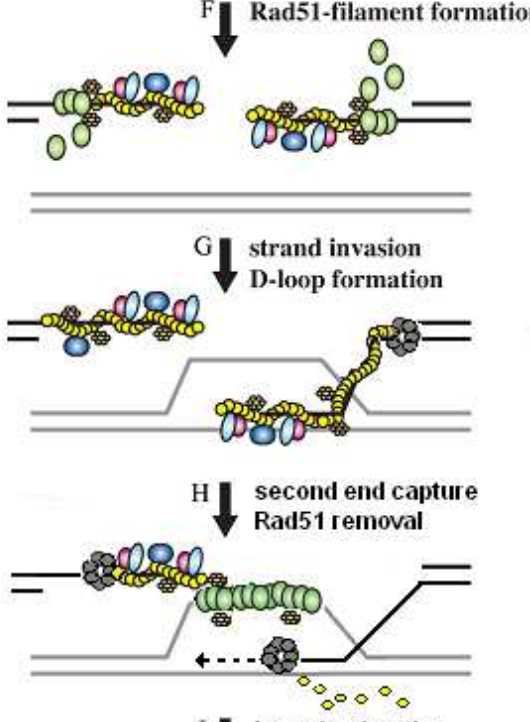

I branch migration

resolution

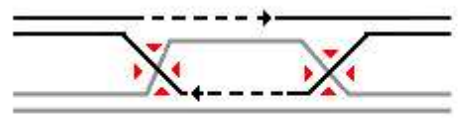

Figure 6 Proteins involved in homologous recombination. The $M R(X) N$ complex serves as a DNA damage sensor and initiates repair. ATM/ATR kinases are recruited to the DSB site and activate checkpoint response. Phosphorylation of surrounding histones amplifies the DNA damage signal. Exonucleases resect the 5'-end of double strand breaks and RPAs and SSBs (single stranded DNA binding proteins) coat the exposed 3'overhangs. Rad51 nucleoprotein filament formation is mediated by many recombination mediators including Rad52, Rad55-57, Hop2-Mnd1 and BRCA2. The nucleoprotein filament mediates strand invasion and D-loop formation. Second-end capture is stimulated by Rad52. Rad54 mediates filament disassembly and DNA polymerase and ligase complete the reaction. Finally, endonucleases cut the Holliday junctions to yield crossover or non-crossover products. (Adapted from Pardo et.al. 2009') 


\section{Discussion}

Genome integrity is essential for the cell's survival, especially during replication. If the DNA is damaged, the cell cannot pass the DNA-damage checkpoint and enters a quiescent state until damage is repaired and genome stability is ensured. Activation of these checkpoints entails complex phosphorylation cascades that trigger numerous protection mechanisms, one of which is homologous recombination. Although the Rad51 nucleoprotein filament mediates all necessary steps for repair, the in vivo situation of course is far more complex. One key event that is still a great mystery is homologous pairing prior to local recognition by the Rad51 filament. Many speculate that pairing between homologous sequences must precede DNA damage in order to account for recombination efficiency in vivo ${ }^{48}$. Sister chromatids are closely localized during meiosis, but recombination also occurs between non-allelic regions. Centromere and telomere clustering of interphase chromosomes may bring homologous regions closer together and decrease the search area. The novel idea of cis-acting pairing centers and specialized repair foci involved in homologous pairing is receiving growing attention. These protein hubs contain large clusters of repair and mediator proteins that co-localize broken DNA molecules for repair.

Single-molecule techniques are becoming increasingly popular to study especially nucleic acid systems. The great disadvantage of these techniques is the highly artificial conditions created in order to perform the experiments. Labeling of proteins with fluorescent dyes can influence function. Methods such as atomic force microscopy or magnetic tweezers require fixation of DNA molecules or attaching one end of the molecule to a glass surfaces. Such interactions may alter the DNA conformation and influence protein binding. Comparison between studies shows that buffer conditions strongly influence the behavior of substrates. Great caution must therefore be taken when extrapolating findings of single-molecule experiments to the in vivo situation.

However, these single-molecule techniques offer some unprecedented benefits. Biological processes commonly progress via a number of structural and functional intermediates that are often obscured in bulk experiments. Single-molecule techniques offer a direct approach to understanding the underlying physical mechanisms and in doing so takes us back to the fundamentals of biology. AFM, magnetic and optical tweezers, in addition, allow manipulation of individual molecules to varying degrees; one example is the famous (over)stretching curves of single DNA molecules ${ }^{25}$. Ultimately, future applications will aim to combine in vivo experiments with single-molecule techniques and allow investigation and manipulation of proteins and molecules inside cells. 


\section{References}

${ }_{1}^{1}$ Pardo B., Gomez-Gonzales B. and Aguilera A., DNA double-strand break repair: How to fix a broken relationship, Cell. Mol. Life Sci. (2009)

${ }^{2}$ Neale M., Pan J. and Keeney S., Endonucleolytic processing of covalent protein-linked DNA double-strand breaks, Nature 436, 1053-1057 (2005)

${ }^{3}$ Tang E. and Martin A., Immunoglobulin gene conversion: Synthesizing antibody diversification and DNA repair, DNA repair 6, 1557-1571 (2007)

${ }^{4}$ Gupta R., Bazemore L., Golub E. and Radding C., Activities of human recombination protein Rad51, Proc. Natl. Acad. Sci. 94, 463-468 (1997)

${ }^{5}$ San Fillippo J., Sung P. and Klein H., Mechanism of eukaryotic homologous recombination, Annu. Rev. Biochem. 77, 9.1-9.29 (2008)

${ }^{6}$ Ritort F., Single-molecule experiments in biological physics: Methods and applications, J. Phys.: Condensed Matter 18, R531-R583 (2006)

${ }^{7}$ Seidel R. and Dekker C., Single-molecule studies of nucleic acid motors, Curr. Opin. Struct. Biol. 17, 80-86 (2007)

${ }^{8}$ Mameren J., Peterman E. and Wuite G., See me, feel me: Methods to concurrently visualize and manipulate single DNA molecules and associated proteins, Nucl. Acids Res. 36, 4381-4389 (2008)

${ }^{9}$ Kowalczykowski S., Dixon A., Eggleston A., Lauder S. and Rehrauer W., Biochemistry of homolgous recombination in Escherichia coli, Microbiol. reviews Vol.58:3 (1994)

${ }^{10}$ Fung C., Fortin G., Peterson S. and Symington L., The rad51-K191R ATPase-defective mutant is impaired for presynaptic filament formation, Molecular and Cellular Biology 26, 9544-9554 (2006)

${ }^{11}$ Lim D. and Hasty P., A mutation in mouse rad51 results in an early embryonic lethal that is suppressed by a mutation in p53, Molecular and Cellular Biology 16, 7133-7143 (1996)

${ }^{12}$ Pellegrini L. et. al., Insights into DNA recombination from the structure of a Rad51-BRCA2 complex, Nature 420, 287-293 (2002)

${ }^{13}$ Thorslund T. and West S., BRCA2: a universal recombinase regulator, Oncogene 26, 7720-7730 (2007)

${ }^{14}$ Cox M., The bacterial RecA protein as a motor protein, Annu. Rev. Microbiol. 57, $551-577$ (2003)

${ }^{15}$ Renodon-Cornière A. et. al., Structural analysis of the human Rad51 protein-DNA complex filament by tryptophan fluorescence scanning analysis: Transmission of allosteric effects between ATP binding and DNA binding, JMB 383, 575-587 (2008)

${ }^{16}$ Grigorescu A. et. al., Inter-subunit interactions that coordinate Rad51's activities, Nucl. Acids Res. 37, 557-567 (2009)

${ }^{17}$ Aihara H., Ito Y., Kurumizaka H., Yokoyama S. and Shibata T., The N-terminal domain of the human Rad51 protein binds DNA: Structure and a DNA binding surface as revealed by NMR, JMB 290, 495-504 (1999)

${ }^{18}$ Conway A. et. al., Crystal structure of a Rad51 filament, Nature Struc. and Mol. Biol. 11, 791-796 (2004)

${ }^{19}$ Yu X., Jacobs S., West S., Ogawa T. and Egelman E., Domain structures and dynamics in the helical filament formed by RecA and Rad51 on DNA, PNAS 98, 8419-8424 (2001)

${ }^{20}$ Galletto R., Amitani I., Baskin R. and Kowalczykowski S., Direct observation of individual RecA filaments assembling on single DNA molecules, Nature 443, 875-878 (2006)

${ }^{21}$ Hilario J., Amitani I., Baskin R. and Kowalczykowski S., Direct imaging of human Rad51 nucleoprotein dynamics on individual DNA molecules, PNAS 106, 361-368 (2009) 
${ }^{22}$ Modesti M. et. al., Fluorescent human Rad51 reveals multiple nucleation sites and filament segments tightly associated along a single DNA molecule, Structure 15, 599-609 (2007)

${ }^{23}$ Joo C. et. al., Real-time observation of RecA filament dynamics with single monomer resolution, Cell 126, 515-527 (2006)

${ }^{24}$ Heijden van der, T. et. al., Real-time assembly and disassembly of human Rad51 filaments on individual DNA molecules, Nucl. Acids Res. 35, 5646-5657 (2007)

${ }^{25}$ McCauley M. and Williams M., Review: Mechanism of DNA binding determined in optical tweezer experiments, Biopolymers 85, 154-168 (2006)

${ }^{26}$ Benson F., Stasiak A. and West S., Purification and characterization of the human Rad51 protein, an analogue of E.coli RecA, EMBO 13, 5764-5771 (1994)

${ }^{27}$ Adzuma K., No sliding during homology search by RecA protein, JBC 273, 31565-31573 (1998)

${ }^{28}$ Granéli A., Yeykal C., Robertson R. and Greene E., Long-distance lateral diffusion of human Rad51 on doublestranded DNA, PNAS 103, 1221-1226 (2006)

${ }^{29}$ Baumann P. and West S., Role of human Rad51 protein in homologous recombination and double-stranded-break repair, TIBS 23, 247-251 (1998)

${ }^{30}$ Cox M., Motoring along with the bacterial RecA protein, Molecular Cell Biology 8, 127-138 (2007)

${ }^{31}$ Heijden van der, $T$ et. al., Homologous recombination in real time: DNA strand exchange by RecA, Molecular Cell 30, 530-538 (2008)

${ }^{32}$ Gupta R., Folta-Stogniew E., O'Malley S. and Takahashi M., Rapid exchange of A:T base pairs is essential for recognition of DNA homology by human Rad51 recombination protein, Molecular Cell 4, 705-714 (1999)

${ }^{33}$ Mameren J. et. al., Counting Rad51 proteins disassembling from nucleoprotein filament under tension, Nature 457, 745-748 (2009)

${ }^{34}$ Buis J. et. al., Mre11 nuclease activity has essential roles in DNA repair and genomic stability distinct from ATP activation, Cell 135, 85-96 (2008)

${ }^{35}$ Moreno-Herrero F. et. al., Mesoscale conformational changes in the DNA-repair complex Rad50/Mre11/Nbs1 upon binding DNA, Nature 437, 440-443 (2005)

${ }^{36}$ Lee J. and Paull T., ATM activation by DNA double-strand breaks through the Mre11-Rad50-Nbs1 complex, Science 308, 551-554 (2005)

${ }^{37}$ Steininger S. et. al., Xrs2 facilitates crossovers during DNA double-strand gap repair in yeast, DNA repair 7, 15631577 (2008)

${ }^{38}$ Celeste A. et. al., Histone H2AX phosphorylation is dispensable for the initial recognition of DNA breaks, Nature Cell Biology Vol. 5:7 (2003)

${ }^{39}$ Strom L., Lindroos H., Shirahige K. and Sjögren C, Postreplicative recruitment of cohesin to double-strand breaks is required for DNA repair, Molecular Cell 16, 1003-1015 (2004)

${ }^{40}$ Nimonkar A., Sica A. and Kowalczykowsky S., Rad52 promotes second-end DNA capture in double-stranded break repair to form complement-stabilized joint molecules, PNAS 106, 3077-3082 (2009)

${ }^{41}$ Fortin G. and Symington L., Mutations in yeast Rad51 that partially bypass the requirement for Rad55 and Rad77 in DNA repair by increasing the stability of Rad51-DNA complexes, EMBO Journal 21, 3160-3170 (2002)

${ }^{42}$ Lio Y., Mazin A., Kowlczykowsky C. and Chen D., Complex formation by the human Rad51B and Rad51C DNA repair proteins and their activities in vitro, JBC 278, 2469-2478 (2003) 
${ }^{43}$ Chi P., San Filippo J., Sehorn M., Petukhova G. and Sung P., Bipartite stimulatory action of the Hop2-Mnd1 complex on the Rad51 recombinase, Genes and Development 21, 1747-1757 (2007)

${ }^{44}$ Mohnahan M., Pierce A. and Jasin M., BRCA2 is required for homology-directed repair of chromosomal breaks, Molecular Cell 7, 263-272 (2001)

${ }^{45}$ Sung P. and Klein H., Mechanism of homologous recombination: mediators and helicases take on regulatory functions, Molecular Cell Biology 7, 739-750 (2006)

${ }^{46}$ Ip S. et.al., Identification of Holliday junction resolvases from human and yeast, Nature 456, 357-362 (2008)

${ }^{47}$ Heyer W., Li X., Rolfsmeier M. and Zhang X., Rad54: the swiss army knife of homologous recombination?, Nucl. Acids Res. 34, 4115-4125 (2006)

${ }^{48}$ Barzel A. and Kupiec M., Finding a match: How do homologous sequences get together for recombination, Genetics 9, 27-37 (2008) 